\title{
Primary Ewing's sarcoma of the orbit presenting with visual loss
}

\author{
G WOODRUFF, ${ }^{1}$ P THORNER, ${ }^{2}$ AND B SKARF
}

'From the Departments of 'Ophthalmology and 'Pathology, the Hospital for Sick Children and University of Toronto, Toronto, Ontario, Canada

SUMMARY A case of primary Ewing's sarcoma of the orbit and paranasal sinuses in a 6-yearold boy is reported. The child presented with headaches and unilateral visual loss. Loss of vision as a result of optic nerve involvement with primary Ewing's sarcoma is extremely rare.

Ewing's sarcoma is a malignant small round-cell tumour typically occurring in the first and second decades of life ${ }^{1}$ and classically involving the long bones of the limbs, the ribs, or the pelvis. ${ }^{2-1}$ Primary Ewing's sarcoma of the head and neck region is unusual and generally involves the mandible or maxilla. ${ }^{5}$ We describe a case involving the orbit and paranasal sinuses in a child with a long history of headache and apparently normal initial findings, who ultimately developed visual loss as a result of optic nerve involvement with primary Ewing's sarcoma.

\section{Case report}

A 6-year-old boy was referred to the Hospital for Sick Children, Toronto, for investigation of headaches and visual loss. His symptoms had started six months previously with a right frontal headache that lasted three days. The headache then recurred every few weeks, lasting up to two hours each time. He was examined on several occasions by his general practitioner, but no abnormality was found. There had been no history of vomiting, nasal obstruction or discharge, systemic symptoms, weight loss, or visual problems at that time. A plain radiograph of the skull taken shortly after the onset of the headaches was reported as normal (Fig. 1A).

Seven days before admission the boy complained of a severe headache and said that his vision was 'going black' in the right eye. Profound visual loss was present in that eye. Repeat plain skull films demonstrated widening of the superior orbital fissure, disruption of the medial third of the sphenoidal ridge, destruction of the planum sphenoidale, and opacifiCorrespondence to Dr G Woodruff, FRCSEd, Tennent Institute of Ophthalmology, Western Infirmary, Glasgow G11 6NT. cation of the right ethmoid sinus (Fig. 1B). The patient was referred to this centre for further examination and management.

On admission, the child was alert, co-operative, and in no distress. Vision in the right eye was limited to bare perception of light temporally. In the left eye the visual acuity was $6 / 6$ and the visual field was full on Goldmann perimetry. There was $2 \mathrm{~mm}$ of right axial proptosis measured with the Hertel exophthalmometer. External examination was otherwise normal. No periorbital masses were palpable round the eye. Ocular motility was unremarkable except for a slight limitation of abduction on the right. The pupils were equal, round, and reactive, but there was a 4 + right relative afferent pupillary defect. Examination of the right fundus revealed a swollen, elevated optic disc with venous engorgement and some loss of the nerve fibre layer. The left fundus was normal.

A computed tomography (CT) scan (Figs. 2A, 2B) showed a large, uncalcified, hyperdense soft-tissue mass involving both ethmoid sinuses, the sphenoid sinus, and the right orbit. The tumour eroded through the planum sphenoidale and the roof of the right orbit, extending superiorly and posteriorly into the anterior and middle cranial fossae. The medial and lateral walls of the right orbit were involved posteriorly, so that the proximal portion of the optic nerve could not be delineated from tumour. More anteriorly there was lateral displacement of the optic nerve and medial rectus muscle associated with extensive destruction of the medial wall of the orbit. The left orbit appeared uninvolved. The tumour showed homogeneous contrast enhancement, with a generally well-defined border in the portion extending intracranially, suggesting that it was extradural.

General examination, ear, nose, and throat exami- 


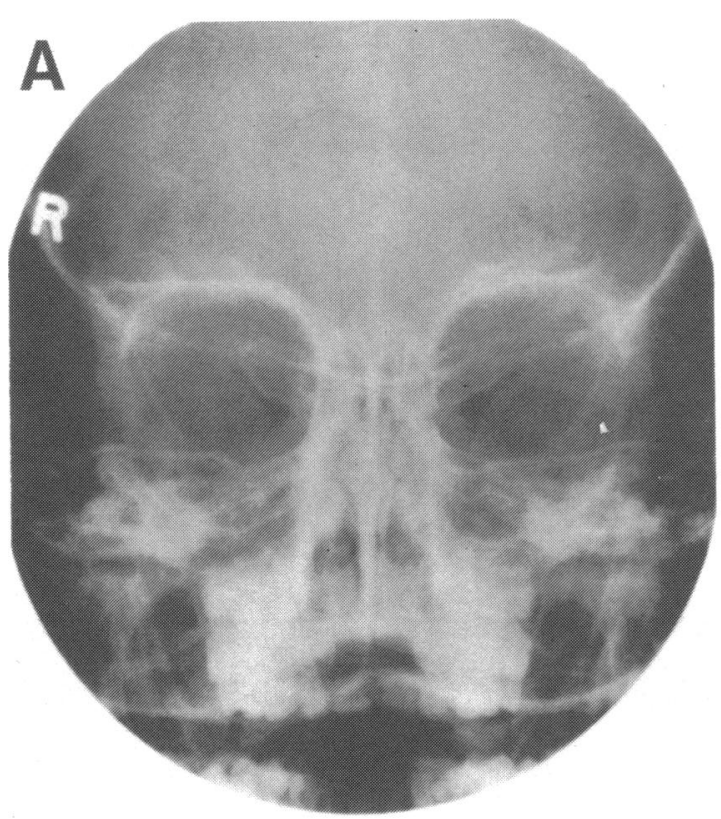

Fig. 1A

Fig. 1 Plain radiographs of the skull. A: Six months before admission. B: At the time of admission for investigation of right visual loss. Note widening of the right superior orbital fissure (arrows).

nation, and neurological assessment were normal. An investigation for metastases, which included bone and gallium scans, a liver-spleen scan, a CT scan of the chest, bone marrow biopsy and aspiration, and examination of cerebrospinal fluid cells failed to demonstrate any other lesion.

An ethmoidectomy and biopsy of tissue from the sphenoid sinus performed under general anaesthesia demonstrated Ewing's sarcoma.

\section{COURSE}

After surgery the child underwent a course of radiation and chemotherapy. 50 Gy targeted to the right orbit and surrounding areas of tumour involvement were administered in 25 fractions, with shielding of the left eye. The patient was prescribed intravenous vincristine, cyclophosphamide, and actinomycin $\mathrm{D}$ as a seven-day course once a month. One dose of cytosine arabinoside, methotrexate, and hydrocortisone was given intrathecally. Six weeks after treatment was started a CT scan showed dramatic shrinkage of the soft-tissue tumour. A CT scan seven months after starting treatment showed healing of the lytic bony lesions, with no recurrence of the tumour mass (Figs. 2C, D).

Nine months after diagnosis the patient continued to receive chemotherapy. He was well and free of

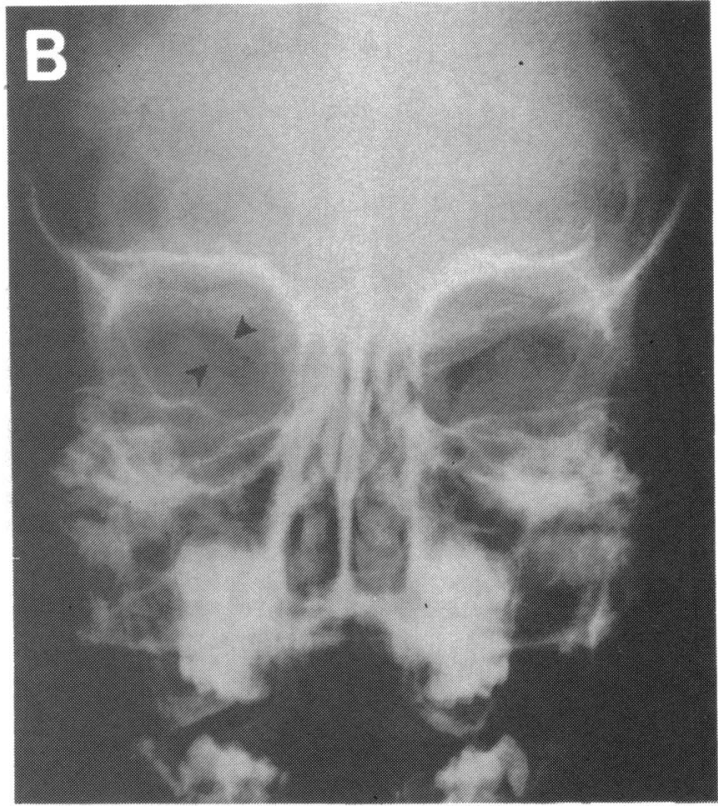

Fig. 1B

headaches. In his right eye there was no light perception and marked optic disc pallor had developed. Visual acuity, visual field, and fundus appearance in the left eye remained normal.

\section{HISTOPATHOLOGY}

Tissue for light microscopy was fixed in $10 \%$ buffered formalin, processed routinely, and embedded in paraffin. Sections were stained with haematoxylin and eosin ( $\mathrm{H}$ and $\mathrm{E}$ ) and periodic acid Schiff (PAS), with and without diastase pretreatment. For immunohistochemistry, sections were stained by the peroxidase-antiperoxidase (PAP) technique using desmin (Euro Diagnostics, Apeldoorn, Netherlands), myoglobin (Miles Scientific, Naperville, Illinois), actin (Dr S. Huang, Sunnybrook Medical Centre, Toronto), S-100 protein (Dakopatts, Glostrup, Denmark), vimentin (BioGenex, Dublin California), neurofilament (Euro Diagnostics), and common leucocyte antigen (Dako).

Tissue for electron microscopy was fixed in $1 \%$ glutaraldehyde and $4 \%$ paraformaldehyde and embedded in Epon-Araldite. Sections were stained with uranyl acetate and lead citrate.

\section{LIGHT MICROSCOPY}

Large clusters and sheets of tumour cells infiltrated bony and soft tissues of the biopsy specimen (Fig. $3 \mathrm{~A}$ ). Tumour cells had uniform round to oval nuclei with dispersed chromatin and occasional nucleoli (Fig. 3B). These cells had a scanty to moderate 


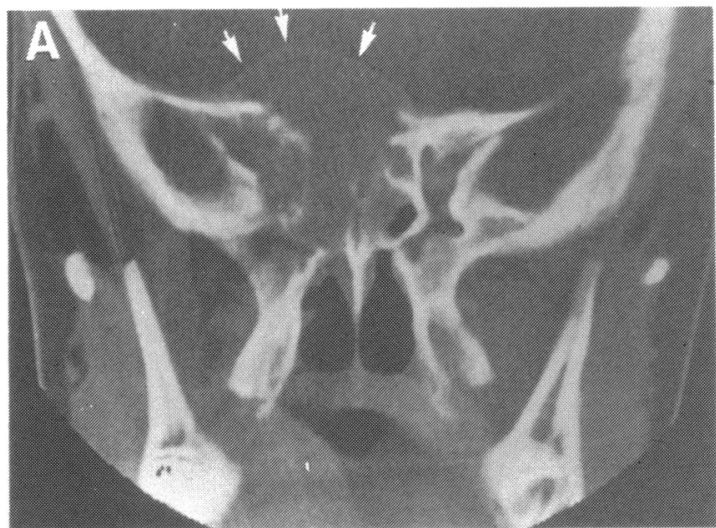

Fig. 2A

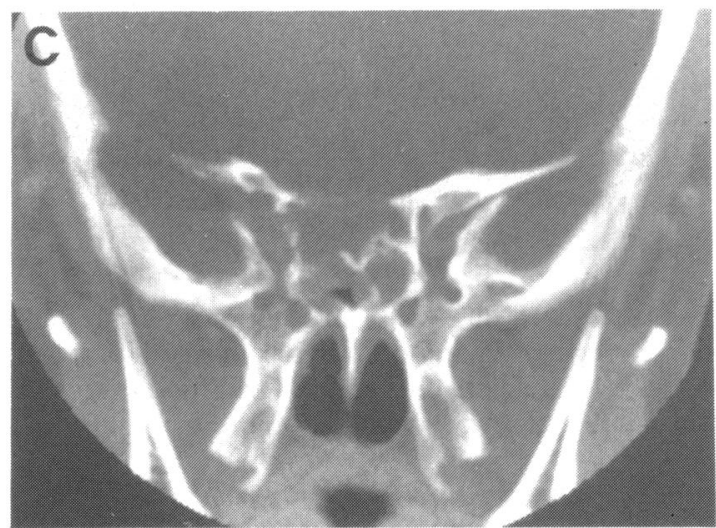

Fig. 2C

Fig. 2 Coronal and axial CT scans of the orbits. A, B: At the time of admission for investigation of right visual loss. Note the well-defined superior border of the tumour (arrows).

C, D: Seven months after starting treatment. Note the resolution of the tumour, with substantial healing of the lytic bonylesions.

amount of vacuolated cytoplasm, which stained for PAS-positive diastase-labile material, indicating the presence of glycogen (Fig. 3C). No evidence of rosette formation or rhabdomyoblastic differentiation was seen.

\section{IMMUNOHISTOCHEMISTRY}

Antibody staining was positive for vimentin only (Fig. 3D). Staining for desmin, myoglobin, and actin (skeletal muscle markers), for neurofilaments, S-100 protein and neuron-specific enolase (neural markers), and for common leucocyte antigen (a lymphoid tissue marker) was negative.

\section{ELECTRON MICROSCOPY}

On electron microscopy the tumour cell nuclei con-

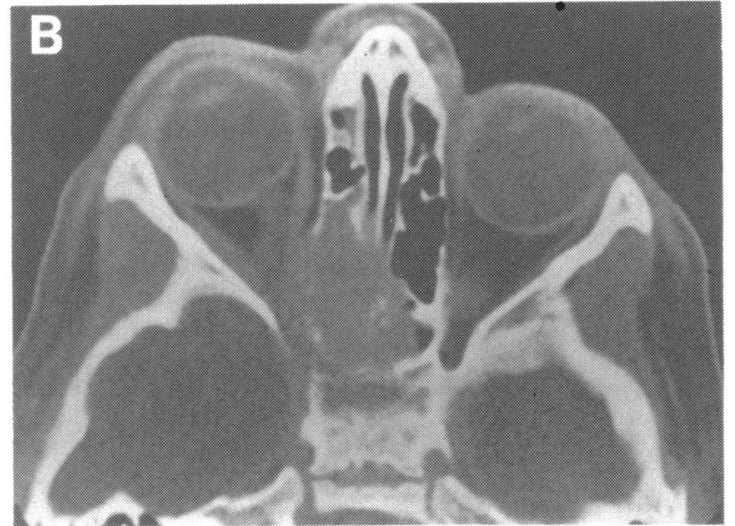

Fig. 2B

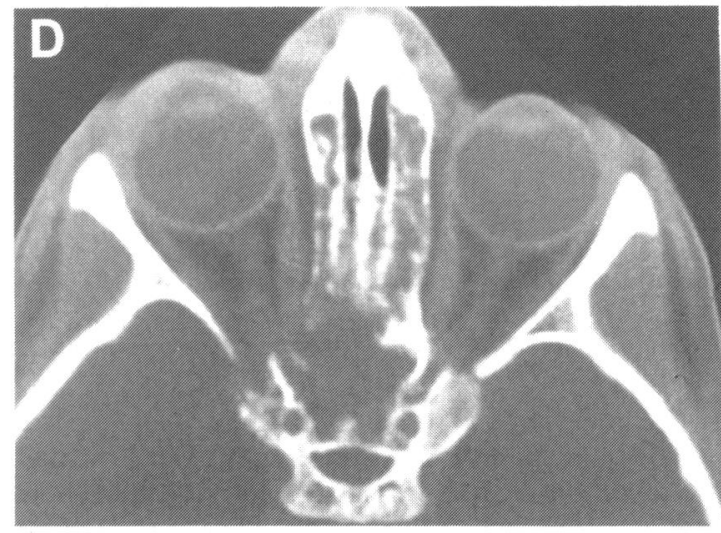

Fig. 2D

tained clumped chromatin at their periphery and a rare nucleolus. The cells possessed a moderate amount of cytoplasm, in which large lakes of glycogen were prominent (Figs. 4A, B). The cytoplasm was relatively simple, with occasional mitochondria and numerous free ribosomes. Occasional tight junctions were identified between tumour cells (Fig. 4C). There was no evidence of neurosecretory granules or neurofilaments. Cytoplasmic processes

Fig 3 A: Photomicrograph of ethmoid bone showing infiltration of marrow spaces by a tumour composed of small round cells. Edges of bony trabeculae show scalloping indicative of resorption. ( $\mathrm{H}$ and $\mathrm{E}, \times 138$ ). $\mathrm{B}$ : Higher magnification of the tumour showing a uniform sheet of small cells with round, bland nuclei and small amounts of clear cytoplasm. (H and E, $\times 553)$. C: tumour cells stained for glycogen, showing abundant deposits within the cytoplasm (arrowheads). (PAS with haematoxylin, $\times 553)$. D: Tumour cells stained for vimentin by immunoperoxidase technique, showing perinuclear staining: nuclear counterstain has been omitted. (Immunoperoxidase, $\times 553$.) 


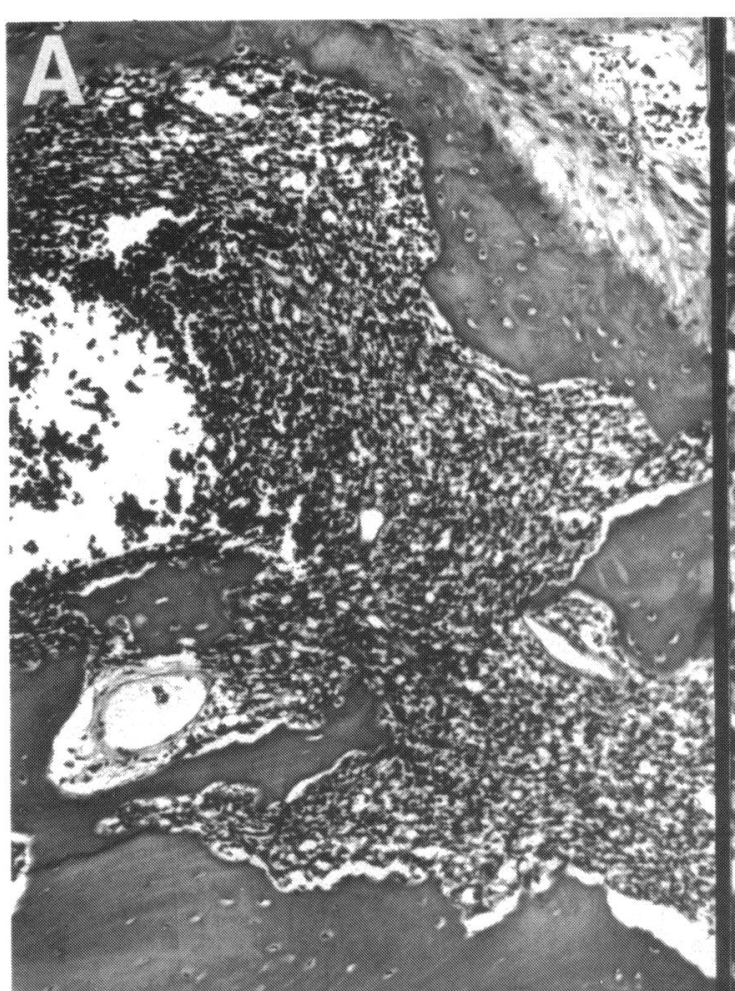

Fig. 3A

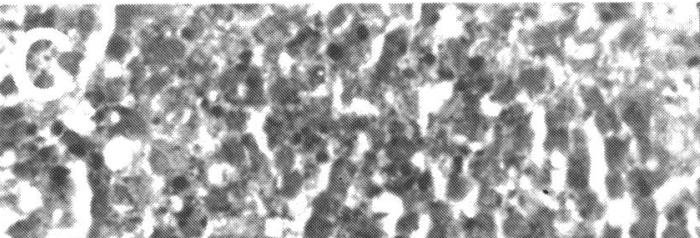

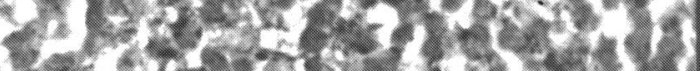

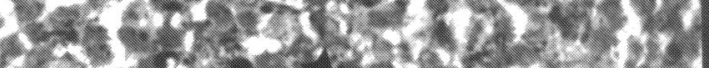

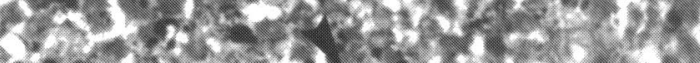

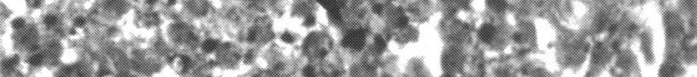

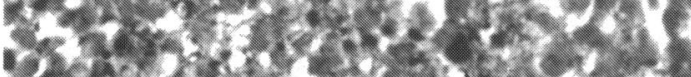
-

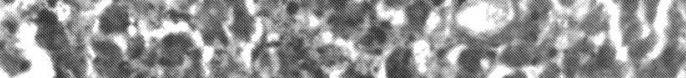

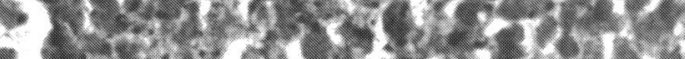

12.

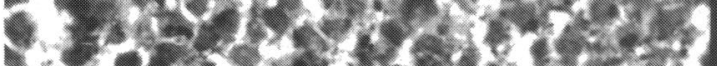

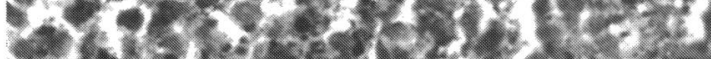

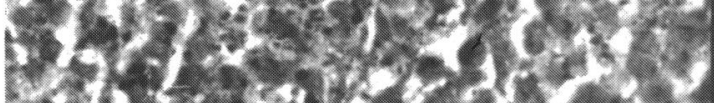

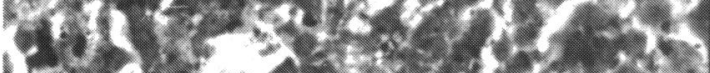
1.

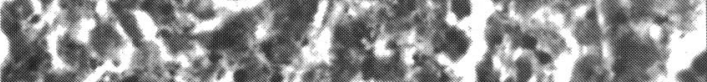

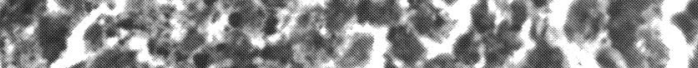

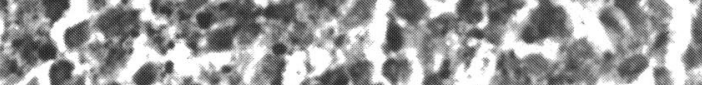

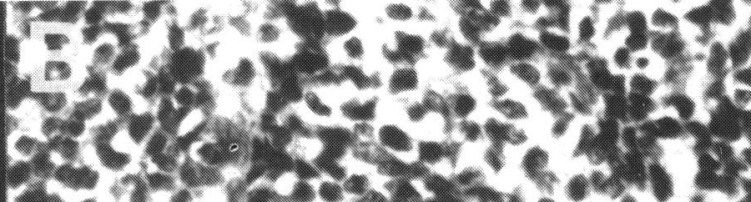

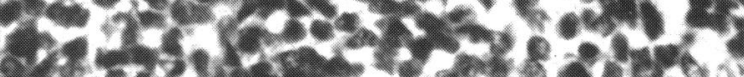

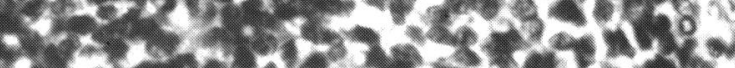

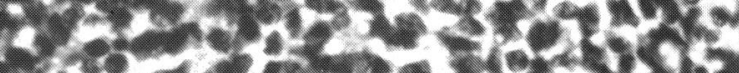

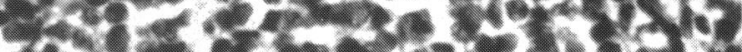

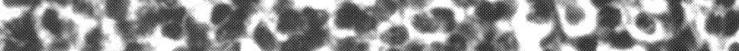

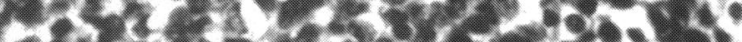

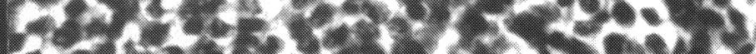

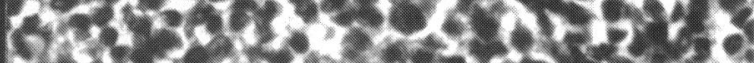

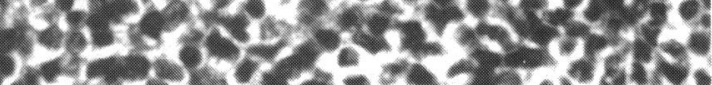

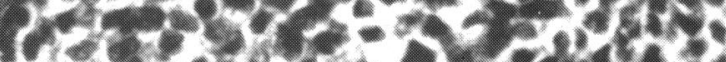

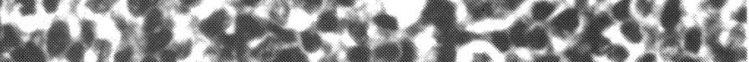

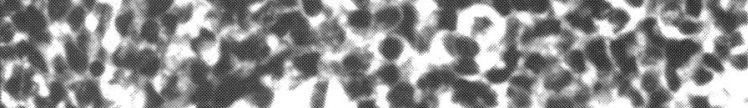

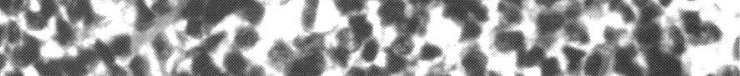

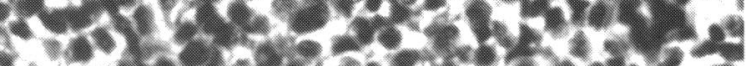

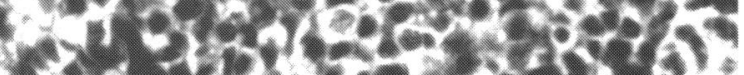

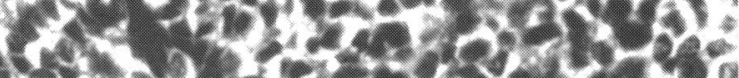

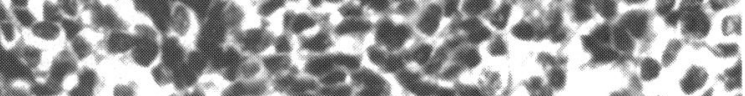

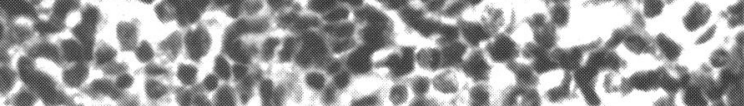
Fig. 3B
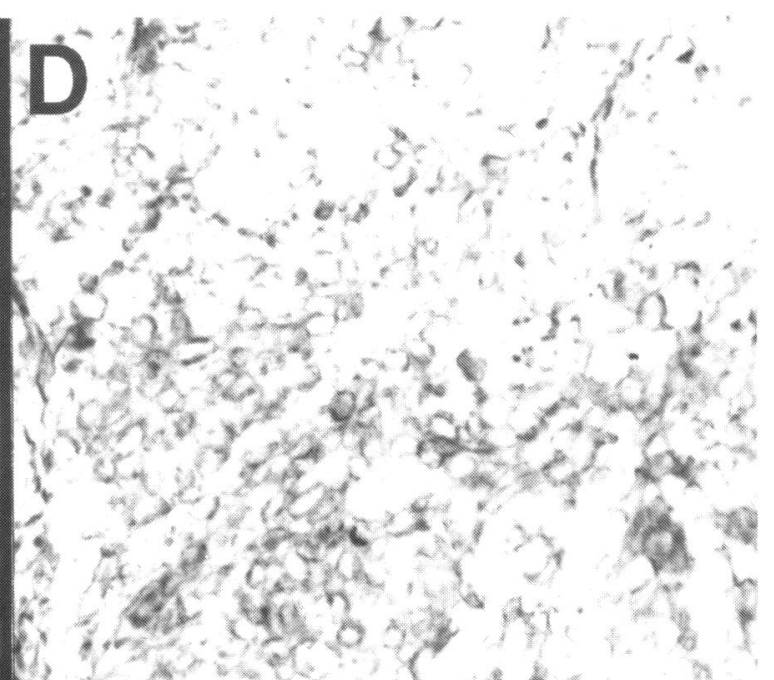

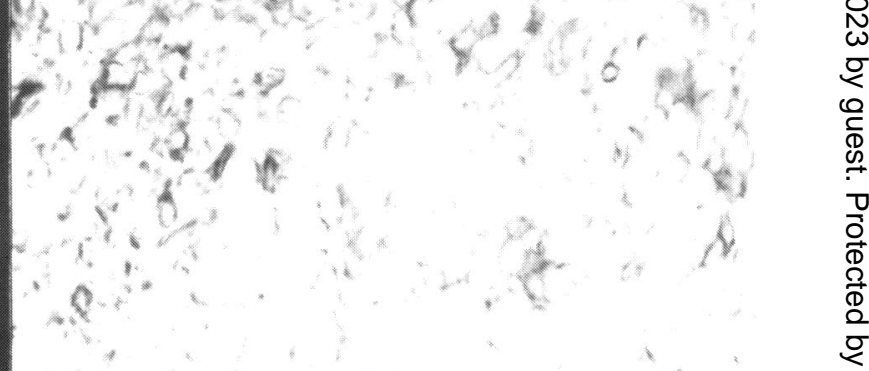




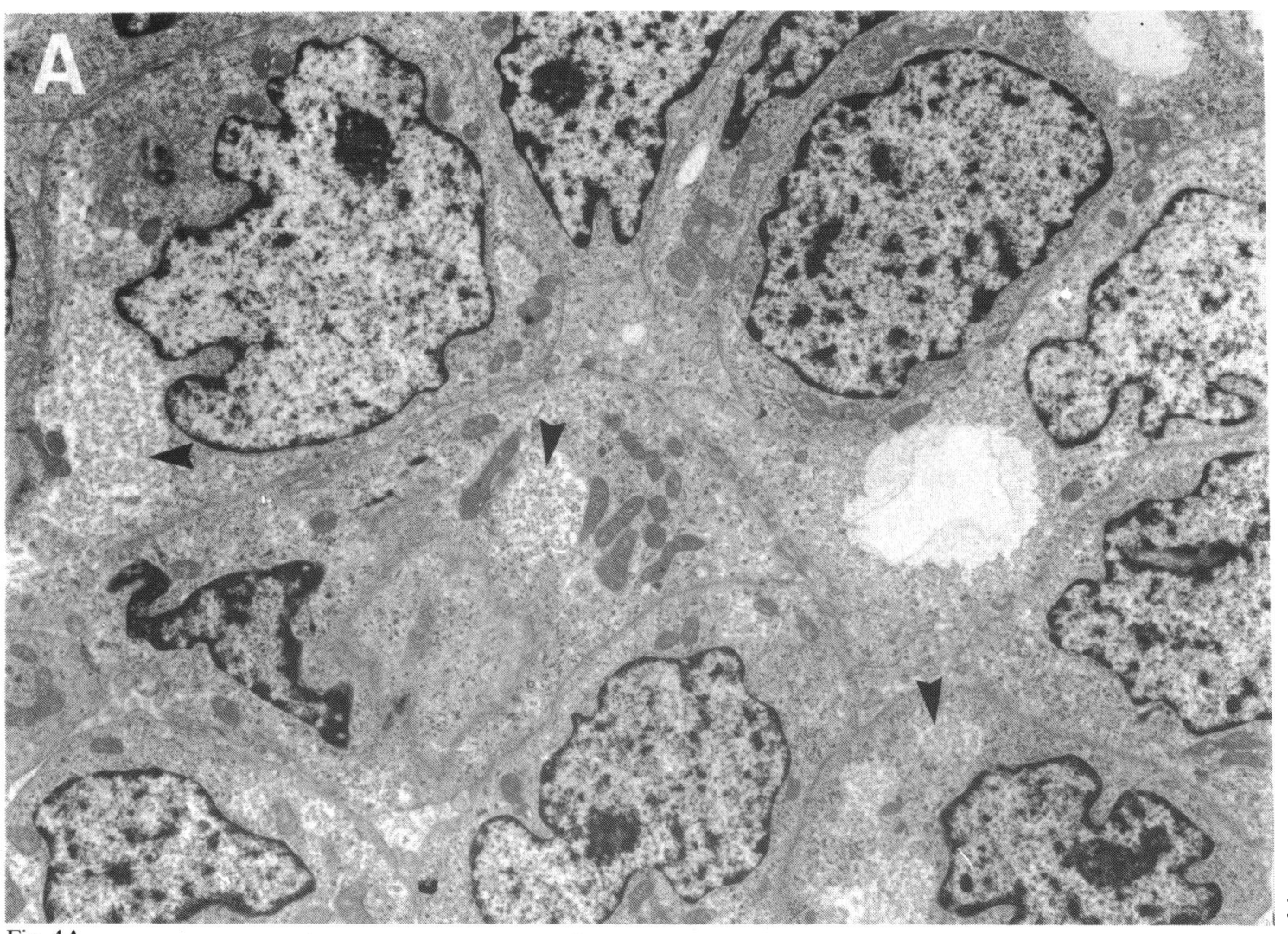

Fig. 4A

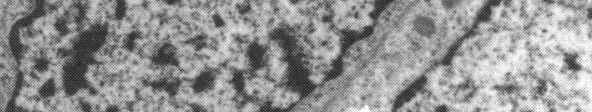

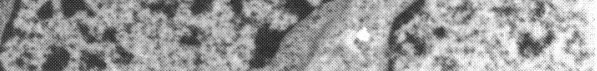

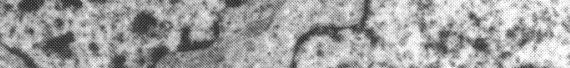

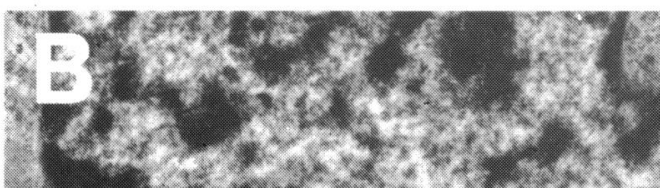

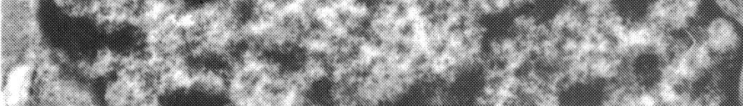

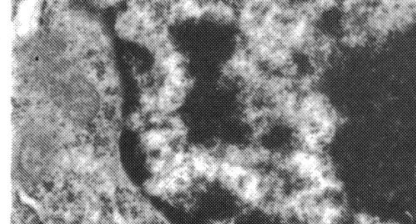

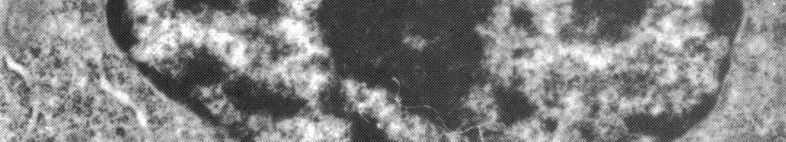

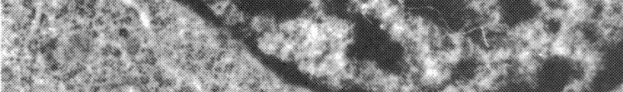

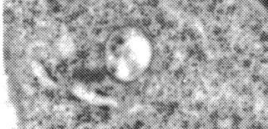

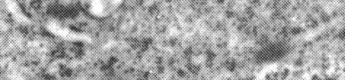

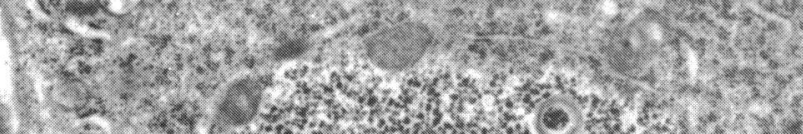

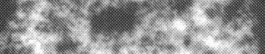

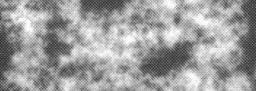

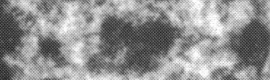

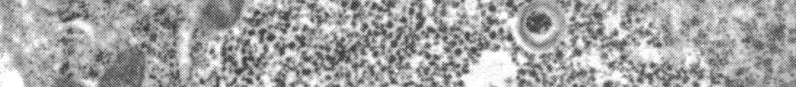

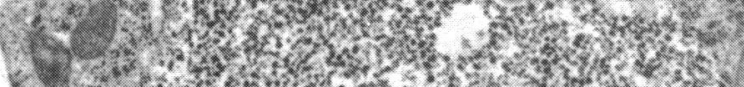

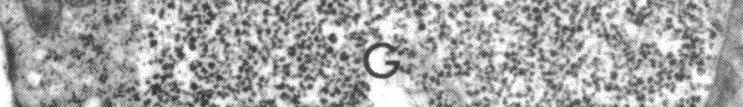

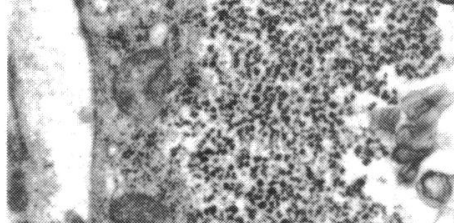

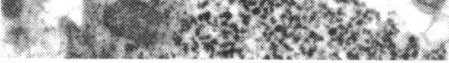

Fig. 4B

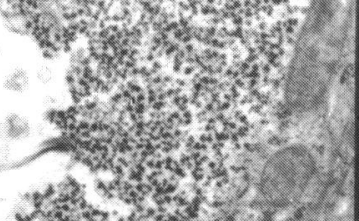

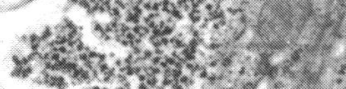

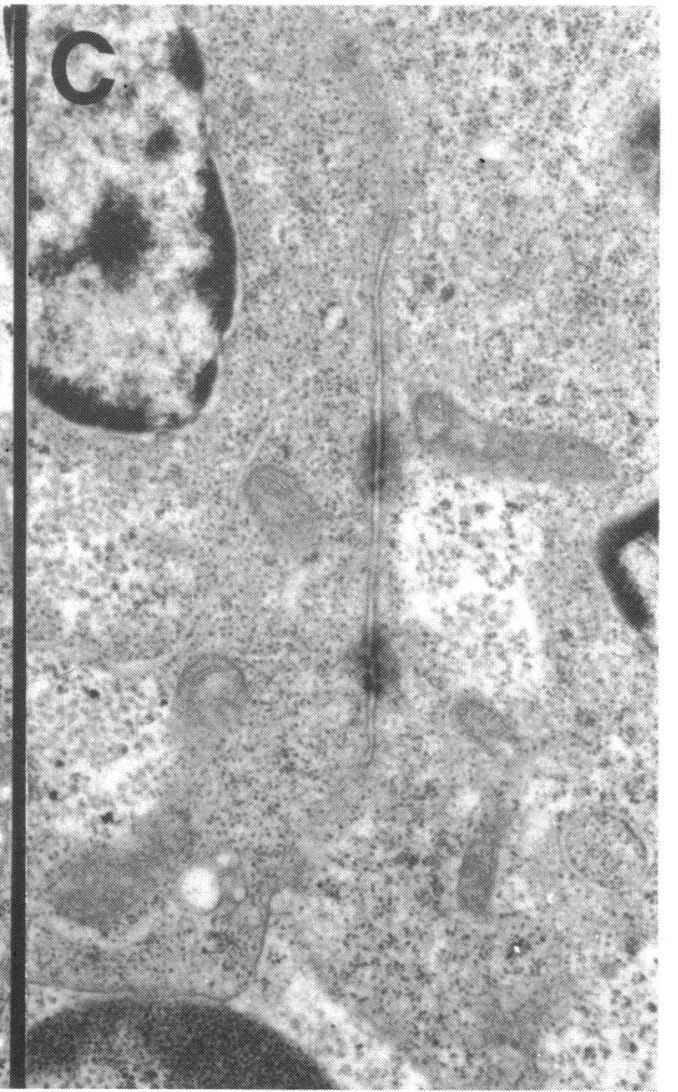

Fig. 4C 
were poorly developed. There were no thick or thin filaments, and no Z-band material was seen.

\section{Discussion}

The history of rapid visual loss, together with optic disc swelling and a small degree of proptosis, in a patient in this age group suggested the possibility of an orbital tumour such as rhabdomyosarcoma. The CT appearance suggested that the large, soft-tissue tumour could be biopsied by infracture of the inferior turbinate, notwithstanding the intact nasal mucosa and clinically normal ENT examination.

Histologically a small round-cell tumour was found. Based on light microscopy, the differential diagnosis at this patient's age included rhabdomyosarcoma, lymphoma, neuroblastoma, and Ewing's sarcoma. Light, immunohistochemical, and electron microscopy demonstrated characteristic features of Ewing's sarcoma, including relatively simple cytoplasm with few intracytoplasmic organelles, the presence of intracytoplasmic glycogen, occasional tight junctions, and positive staining for vimentin. Cell markers and ultrastructural features of neural, skeletal, and lymphoid tumours were absent. The thorough search for another primary site was negative, confirming that the tumour was a primary Ewing's sarcoma of the right orbit and paranasal sinuses.

Orbital Ewing's sarcomas are usually metastases from distant sites ${ }^{67}$ In Coley and colleagues ${ }^{1}$ review of 91 patients with primary Ewing's sarcoma seen over a 30-year period there was no patient with a primary tumour in the head and neck. More recently primary tumours in this region have been recognised as accounting for $2-3 \%$ of all Ewing's sarcomas, with most of these affecting the mandible. ${ }^{58}$ The previously reported cases of primary Ewing's sarcoma arising in the orbital region have presented with a visible exostosis" or a massive intracranial tumour ${ }^{11}$ " or both. ${ }^{12} 13$

Harbert and Tabor ${ }^{13}$ described a 19 -year-old man who presented with temporal fossa swelling, gross proptosis, and diplopia, but minimal impairment of vision. His massive tumour originating in the temporal fossa was demonstrated histologically to be Ewing's sarcoma, and it proved fatal within a few months despite radiotherapy. Yamada and Taka-

Fig. 4 A: Electron micrograph showing tumour composed of cells with round to slightly convoluted nuclei, occasional nucleoli, and occasional cytoplasmic organelles, mainly mitochondria and free ribosomes. Large glycogen lakes (arrowheads) are prominent. $(\times 7000$.) B: Higher power of a single tumour cell showing cytoplasmic glycogen deposits (G). $(\times 10500$.$) C: Portions of two tumour cells showing$ occasional cell junction formations. $(\times 14875$.) hashi" described the post-mortem pathology of a patient with a large intra- and extracranial primary Ewing's sarcoma that involved the orbital region. Alvarez-Berdecia et al. ${ }^{12}$ reported on a 6-year-old boy who presented with headaches and a rapidly growing tender exostosis on his forehead. He had papilloedema associated with a massive intracranial tumour arising from the supraorbital region and occupying most of the anterior cranial fossa. Vision in the eye was 20/50 (6/15). Beraud and Fortin" described two cases of Ewing's sarcoma with intracranial extension and papilloedema but apparently no direct involvement of the optic nerve. Both of these patients died within three weeks of presenting with a bony swelling in the temporal region. More recently Howard and Lund" reported on a patient who presented with an exostosis palpable over the nasal bridge and apparently normal visual function; he had a primary Ewing's sarcoma of the ethmoid sinus.

Our patient's presentation differed substantially from those described previously in the literature. This boy had no externally visible lesion and minimal proptosis. His principal complaints were headache and profound visual loss. To the best of our knowledge this is the first case of a primary Ewing's sarcoma presenting with substantial visual loss.

Small round-cell tumours of the orbital region producing visual loss are not rare in the first and second decades of life, ${ }^{6-1+}$ but typically they are lymphomas or rhabdomyosarcomas. When poorly differentiated they can be distinguished from Ewing's sarcoma only by immunohistochemical and electronmicroscopic techniques that have become widely available only relatively recently. Thus previous surveys of orbital and paranasal tumours may have included some unrecognised Ewing's sarcomas. If this is the case, primary Ewing's sarcoma of this region, including cases with visual loss, may be recognised more frequently in future.

This work was supported in part by the Greater Glasgow Health Board and the Keeler Foundation for Ophthalmology. The authors thank Dr Helen Chan for details of this patient's treatment, Dr Patricia Burrows and the Division of Neuroradiology for radiological advice, and Carla Salvador of the Medical Publications Department for assistance in preparing the manuscript.

\section{References}

1 Coley BL, Higinbotham NL, Bowden L. Endothelioma of bone (Ewing's sarcoma). Ann Surg 1948; 128: 533-60.

2 Ewing J. Diffuse endothelioma of bone. Proc NY Pathol Soc 1921; $21: 17-24$.

3 Jereb B, Ong RL, Mohan M, Caparros B, Exelby P. Redefined role of radiation in combined treatment of Ewing's sarcoma. Pediatr Hematol Oncol 1986; 3: 111-8.

4 Senac MO Jr, Isaacs H, Gwinn JL. Primary lesions of bone in the 1st decade of life: retrospective survey of biopsy results. Pediatr Radiol 1986; 160: 491-5. 
5 Wilkins RM, Pritchard DJ, Burgert EO Jr, Unni KK. Ewing's sarcoma of bone. Experience with 140 patients. Cancer 1986; 58: 2551-5.

6 Blodi FC. Pathology of orbital bones. Am J Ophthalmol 1976 ; 81: 1-26.

7 Jacobiec FA, Font RL. Metabolic orbital tumors in children. In: Spencer WH, ed. Ophthalmic pathology: a textbook and atlas. Philadelphia: Saunders, 1985: 2749-50.

8 Dahlin DC. Coventry MB, Scanlon PW. Ewing's sarcoma. A critical analysis of 165 cases. J Bone Joint Surg 1961; 43A: 185-92.

9 Howard S, Lund VJ. Primary Ewing's sarcoma of the ethmoid bone. J Laryngol Otol 1985; 99: 1019-23.

10 Yamada S, Takahashi H. A dissected case of Ewing's sarcoma (reticulum cell sarcoma) of the orbit. Hirosaki Med J 1957; 8: $202-7$.

11 Beraud R, Fortin P. Sarcome d'Ewing à localisation temporale. Can Med Assoc J 1967; 97: 338-41.

12 Alvarez-Berdecia A, Schut L, Bruce DA. Localized primary intracranial Ewing:s sarcoma of the orbital roof. Case report. $J$ Neurosurg 1979; 50: 811-3.

13 Harbert F. Tabor GL Jr. Ewing's tumor of the orbit. Am J Ophthalmol 1950; 33: 1219-55.

14 Knowles DM II, Jakobiec FA, Potter GD, Jones IS. Ophthalmic striated muscle neoplasms. Surv Ophthalmol 1976; 21:219-61.

Accepted for publication 17 July 1987. 